\title{
NON-EXISTENCE RESULTS AND ANALYTICAL BOUNDS OF EIGENVALUES FOR A CLASS OF FRACTIONAL EIGENVALUE PROBLEMS
}

\author{
MOHAMmed AL-REFAI
}

Abstract. In this paper, we study a class of linear and nonlinear fractional eigenvalue problems with fractional derivative of Caputo type. The problem is obtained by fractionalizing a term of the well-known Sturm-Liouville operator and it covers a wide class of fractional eigenvalue problems. By applying simple maximum principles, we obtain necessary conditions for the existence of eigenfunctions and analytical bounds for the eigenvalues. We also establish a uniqueness result for the nonlinear eigenvalue problem. The results in this paper are discussed in two common spaces of fractional derivatives.

Mathematics subject classification (2010): 34A08, 34Bxx, 35J40.

Keywords and phrases: Fractional differential equations, Caputo fractional derivative, maximum principles, Sturm-Liouville eigenvalue problems.

\section{REFERENCES}

[1] Arwa Ba Abdulla, M. Al-Refai And A. Al-Rawashdeh, On the Existence and Uniqueness of Solutions for a Class of non-Linear Fractional Boundary Value Problems, Journal of King Saud University-Science, 28 (2016), 103-110.

[2] T. S. Aleroev, H. T. Aleroeva, A problem on the zeros of the Mittag-Liffler function and the spectrum of a fractional-order differential operator, Electronic Journal of Qualitative Theory of Differential Equations 25 (2009), 1-18.

[3] H. T. Aleroeva, T. S. Aleroev, N.-M. Nie, Y.-F. TAng, Boundary value problems for differential equations of fractional order, Memoirs on Differential Equations and Mathematical Physics 49 (2010), 21-82.

[4] M. AL-REFAI, Basic results on nonlinear eigenvalue problems of fractional order, Electronic Journal of Differential Equations Vol. 191 (2012), 1-12.

[5] M. Al-Refai, T. Abdeljawad, Fundamental Results of Conformable Sturm-Liouville Eigenvalue Problems, Complexity, Volume 2017 (2017), Article ID 3720471, 7 pages, https://doi.org/10.1155/2017/3720471.

[6] Q. Al-Mdallal, An efficient method for solving fractional Sturm-Liouville problems, Chaos, Solitons and Fractals 1, 40 (2009), 183-189.

[7] P. Antunes, R. FERREIRA, An augmented-RBF method for solving fractional Sturm-Liouville eigenvalue problems, Siam Journal of Scientific Computing, Jan. 2015, DOI 10.1137/140954209.

[8] L. Changpin, D. Weinau, Remarks on fractional derivatives, Appl. Math. Comput. 187 (2007), $777-784$.

[9] V. ERTÜRK, Computing eigenelements of Sturm-Liouville problems of fractional order via fractional differential transform method, Mathematical and Computational Applications 3, 16 (2011), 712-720.

[10] M. A. Hajui, Q. Al-Mdallal, F. Allan, An efficient algorithm for solving higher-order fractional Sturm-Liouville eigenvalue problems, Journal of Computational Physics 272 (2014), 550-558.

[11] Z. HAN, J. LiU, S. SUn, Y. ZHAO, Eigenvalue problem for a class of nonlinear fractional differential equations, Ann. Funct. Anal. 1, 4 (2013), 25-39.

[12] B. Jin, R. Lazarov, J. Pasciak, W. Rundell, A finite element method for fractional SturmLiouville problem, arXiv:1307.5114v1[Math.NA], 2013. 
[13] M. Klimek and O. Agrawal, Fractional Sturm-Liouville problem, Comput. Math. Appl. 5, 66 (2013), 795-812.

[14] M. KLIMEK, Fractional Sturm-Liouville problem in terms of Riesz derivatives, Theoretical development and applications of non-integer order systems, 7th Conference on Non-Integer Order Calculus and its Applications, Szczecin, Ploand, 2015.

[15] J. LI, J. QI, Spectral problems for fractional differential equations from nonlocal continuum mechanics, Advances in Difference Equations 2014, 85 (2014), doi:10.1186/1687-1847.

[16] I. Podlubny, Fractional Differential Equations, Academic Press, New York, 1999.

[17] K. PRASAD, B. KRUSHNA, Eigenvalues for iterative systems of Sturm-Liouville fractional order twopoint boundary value problems, Fractional Calculus and Applied Analysis 3, 17 (2014), 638-653.

[18] M. Rivero, J. Trujillo, M. Velasco, A fractional approach to the Sturm-Liouville problem, Cent. Eur. J. Phys. 11 (2013), 1246-1254.

[19] V. KiRYA KovA, Generalized Fractional Calculus and Applications, John Wiley and Sons, New York, 1994.

[20] W. WANG AND X. Guo, Eigenvalue problem for fractional differential equations with nonlinear integral and disturbance parameter in boundary conditions, Bound Value Probl. 42 (2016), doi:10.1186/s13661-016-0548-0.

[21] G. Zayernouri, G. KARniadakis, Fractional Sturm-Liouville eigen-problems: Theory and numerical approximation, Journal of Computational Physics 252 (2013), 495-517. 